\title{
PERFIL DE ATENDIMENTO REMOTO A CRIANÇAS COM DESORDENS NEURO- LÓGICAS NA REDE APAE DURANTE A PANDEMIA DA COVID-19 UM ESTUDO DO TIPO SURVEY
}

\author{
Maria Eduarda Silva Mendes ${ }^{1}$ \\ Suiani da Silveira ${ }^{2}$ \\ Hanahira dos Santos Machado ${ }^{3}$ \\ André Luís Ferreira Meireles ${ }^{4}$
}

Resumo: Devido à adoção de medidas de isolamento e de distanciamento social como meios de controlar a disseminação da Covid-19, diversas profissões da área da saúde tiveram que se adaptar ao novo cenário. Assim, como forma de manter a assistência, mesmo que de forma remota, foi aprovado o uso do telemonitoramento por fisioterapeutas. Dessa forma, o presente estudo tem como objetivo reportar as características do telemonitoramento adotado por fisioterapeutas às crianças com desordens neurológicas vinculadas à Rede de Associação de Pais e Amigos dos Excepcionais (APAE). Este trabalho é caracterizado como um estudo transversal do tipo survey, realizado a partir de um questionário eletrônico a fisioterapeutas neuropediátricos brasileiros constituintes da REDE APAE. O questionário da pesquisa foi composto por duas partes principais: a) dados demográficos e profissionais e b) questões sobre as características da assistência de telemonitoramento. No total, 318 fisioterapeutas responderam ao questionário, a maioria mulher $(84,34 \%)$ e da região sudeste do país $(46,67 \%)$. O número de atendimentos semanais foi menor no período pré-pandemia (30 ou mais atendimentos $(35,44 \%)$ quando comparado ao período da pandemia (6 a 10 crianças $(21,38 \%$ ). Os recursos mais utilizados para auxiliar no atendimento foram o uso de bolas, rolos e bancos $(69,11 \%)$. Tratando-se de questões referentes às principais dificuldades reportadas pelos fisioterapeutas houve maior destaque quando à falta de compreensão por pais ou responsáveis em manuseios específicos e problemas de conexão com a internet. Este estudo adiciona informações importantes sobre o uso da telerreabilitação pela fisioterapia na população neuropediátrica. Dessa forma, os resultados são úteis para o norteamento, bem como auxílio na elaboração e adequações das consultas via telemonitoramento, por profissionais e tomadores de decisão na área da reabilitação pediátrica.

Palavras-chave: Paralisia cerebral. Fisioterapia neuropediátrica. Telerreabilitação.

\footnotetext{
Abstract: Because of isolation and social distancing measures to control the spread of Covid-19, several healthcare professionals had to adapt to the new scenario. Thus, to maintain care, even if remotely, the use of telemonitoring by physical therapists was approved. Because of this, this study aims to report the characteristics of telemonitoring adopted by physical therapists for children with neurological disorders linked to the Associação de Pais e Amigos dos

1 Centro Universitário Unifacvest.

2 Centro Universitário Unifacvest.

3 Centro Universitário Unifacvest.

4 Centro Universitário Unifacvest; Universidade do Estado de Santa Catarina.
} 
Excepcionais - APAE (Parents and Friends of People with Special Needs Association) Network. The study is characterized as a cross-sectional survey-type study, carried out from an electronic questionnaire to Brazilian neuropediatric physiotherapists who are members of the APAE. The survey questionnaire comprised two main parts: (1) demographic and professional data and (2) questions about the characteristics of telemonitoring help. In total, 318 physical therapists answered the questionnaire, mostly women (84.34\%) and from the Southeast region of the country $(46.67 \%)$. The number of weekly visits decreases in the pre-pandemic period (30 or more visits $(35.44 \%)$ ) when compared to the pandemic period (6 to 10 children $(21.38 \%)$ ). The most used resources to assist with care were the use of balls, rollers, and benches (69.11\%). Regarding issues related to the major difficulties reported by physical therapists, there was a greater emphasis on the lack of understanding by parents or guardians in specific handling and internet connection problems. This study adds important information about the use of telerehabilitation by physical therapy in the neuropediatric population. Thus, being useful as guidance in the preparation and adaptation of consultations via telemonitoring by professionals and decision-makers in pediatric rehabilitation.

Keywords: Cerebral palsy. Neuropediatric physical therapy. Telerehabilitation.

\section{INTRODUÇÃO}

A pandemia da Covid-19 teve o seu início em meados de março de 2020. Em poucos meses, o vírus se disseminou por diversos países, incluindo o Brasil (MARINHO et al., 2020). Devido à velocidade de propagação e ao número crescente de infectados e óbitos, a Organização Mundial de Saúde (OMS) recomendou que medidas, como uso de máscaras, distanciamento e isolamento social fossem implementadas, para diminuir as taxas de contágio viral (ORGANIZAÇÃO MUNDIAL DA SAÚDE, 2020).

As recomendações da OMS foram adotadas por diversos países e outras medidas também foram utilizadas pelos governantes, como campanhas sobre higiene, limitação da circulação de pessoas e restrição de serviços não essenciais (ORGANIZAÇÃO MUNDIAL DA SAÚDE, 2020; HAN et al., 2020). Tendo em vista as medidas empregadas logo no início da pandemia e a incerteza sobre essa nova doença, algumas profissões da área da saúde se adaptaram ao novo cenário, permitindo o atendimento não presencial.

A fisioterapia foi uma das profissões que aderiu ao atendimento pela modalidade remota durante o período pandêmico. Pela resolução ${ }^{\circ}$ 516, publicada em março de 2020, o Conselho Federal de Fisioterapia e Terapia Ocupacional (COFFITO) aprovou três modalidades de assistências remota durante a pandemia: a teleconsulta, a qual está relacionada com o atendimento não presencial no primeiro contato entre terapeuta e paciente; o telemonitoramento, o qual é definido como atendimento a distância ao paciente que já fora atendido previamente e presencialmente pelo terapeuta e, por fim, a teleconsultoria, que consiste na comunicação entre profissionais de forma remota, a fim de esclarecer dúvidas sobre procedimentos clínicos (COFFITO, 
2020).

A alternativa da telerreabilitação para os fisioterapeutas foi uma medida importante, tendo em vista as restrições impostas, segurança dos profissionais, pacientes e familiares, bem como a continuidade do direito à saúde da pessoa com deficiência (ADERONMU, 2020). Dentre as diferentes populações atendidas pela fisioterapia, a pediátrica seria talvez a que menos sofresse com a pandemia, tendo em vista que durante esse período se observou que as crianças apresentam uma melhor imunidade para essa nova doença (DHOCHAK et al., 2020). Entretanto, quando se pensa no perfil de pacientes atendidos em organizações como a Associação de Pais e Amigos dos Excepcionais (APAE), no qual observamos crianças com doenças neurológicas e deficiências intelectuais que podem apresentar diversas condições respiratórias prévias, a população pediátrica acaba por se tornar mais vulnerável pelo seu quadro clínico de base (ORGANIZAÇÃO MUNDIAL DA SAÚDE, 2020; MEIRELES; MEIRELES, 2020; HULS et al., 2021). Dessa forma, o atendimento fisioterapêutico não presencial ofertado pela REDE APAE oferecido para essa população se torna extremamente importante, mas também desafiador.

O atendimento remoto voltado para a população neuropediátrica vem sendo demonstrado em diversos estudos. Esses, por sua vez, reportam a presença de dificuldades e desafios do atendimento não presencial, sendo evidente: a necessidade de um adulto responsável presente durante a sessão, problemas com conexão de internet e ausência de materiais específicos, dificultando a entrega e execução do tratamento fisioterápico eficiente (MARESCA et al., 2020; TENFORDE et al., 2020; CAMDEN; SILVA, 2019).

Tendo em vista esse panorama desafiador imposto pelo novo Coronavírus aos fisioterapeutas neuropediátricos, o objetivo desse estudo foi descrever as características do atendimento fisioterapêutico em crianças com condições neurológicas por telemonitoramento, durante a pandemia de Covid-19.

\section{METODOLOGIA}

\section{Design de estudo}

Foi realizado um estudo transversal do tipo survey, com fisioterapeutas brasileiros que prestaram assistência por telemonitoramento a crianças e adolescentes com desordens neurológicas durante a pandemia de Covid-19. A presente pesquisa foi aprovada pelo Comitê de Ética em Pesquisa do Centro Universitário Unifacvest, pelo protocolo CAAE: 39421720.4.0000.5616.

\section{Participantes}

Os fisioterapeutas incluídos neste estudo corresponderam a profissionais vinculados à Associação de Pais e Amigos dos Excepcionais (APAE), que aderiram ao atendimento remoto na modalidade de telemonitoramento em crianças, com disfunções neurológicas, devidamente 
registrados e ativos em seus respectivos Conselhos Regionais de Fisioterapia e Terapia Ocupacional (CREFITO).

O recrutamento dos fisioterapeutas ocorreu no mês de setembro de 2020, sendo a participação dos profissionais voluntária, com consentimento fornecido por um termo livre e esclarecido contido no início do questionário on-line. Com o intuito de demonstrar a realidade das diversas regiões brasileiras, a divulgação e a distribuição de um link correspondente a um questionário eletrônico via Google Forms ${ }^{\circledR}$ foi realizada em parceria com a Federação Nacional das APAES (FENAPAES).

\section{Procedimentos}

Foi desenvolvido um questionário eletrônico via plataforma Google Forms ${ }^{\circledR}$, cuja composição partiu do embasamento de estudos publicados anteriormente sobre telessaúde (AYATOLLAHI et al., 2015; PEEK et al., 2016) e uma diretriz abordando o respectivo tema (LANGBECKER et al., 2017). Para a elaboração final do questionário eletrônico, duas revisões foram efetuadas por dois fisioterapeutas neuropediátricos experientes, sendo atribuídas ao estudo todas as recomendações sugeridas para a versão final do questionário (Apêndice).

O questionário foi composto pelas seguintes seções: a) exposição breve do objetivo e finalidade da pesquisa, procedimentos e termo de consentimento livre e esclarecido; b) aspectos sociodemográficos e referentes ao perfil dos profissionais, como sexo, idade, ano de conclusão da graduação, cursos de especialização na área neuropediátrica e local de assistência fisioterapêutica e c) questões específicas sobre a assistência do fisioterapeuta durante o período de isolamento social ocasionado pela Covid-19, relatando aspectos quanto à quantidade de crianças atendidas antes e após a pandemia, duração do atendimento, impacto nos honorários cobrados, método utilizado para disponibilizar o telemonitoramento, instrumentos empregados durante a intervenção, treinamento prévio dos profissionais, principais dificuldades, entre outros pontos (AYATOLLAHI et al., 2015) (Apêndice).

\section{Análise de dados}

Após o período de recrutamento dos profissionais, os dados obtidos foram exportados do formulário de pesquisa e importados para análise em software estatístico específico (IBM SPSS Versão 22.00). Essas informações foram realizadas de forma descrita e expostas em frequência e porcentagem.

\section{RESULTADOS}

Registrou-se pelo formulário eletrônico 436 respostas. Dessas, 30 não foram incluídas, devido ao critério exigido de ser fisioterapeuta. Além disso, houve a necessidade da exclusão de 
quatro resolutivas duplicadas e 84 respostas daqueles que afirmaram não ter atendido crianças pelo formato telemonitoramento, resultando, a partir disso, em 318 participantes com respostas válidas.

\section{Dados sociodemográficos}

A partir da análise dos dados, verificou-se que a maior parte da população amostral foi composta pelo sexo feminino $(265,84,39 \%)$, da região Sudeste $(147,46,67 \%)$, com idades entre 22 e 63 anos $(35,80 \pm 7,61)$, apresentando a conclusão da graduação entre os anos de 2000 e 2010 $(154,49,68 \%)$. Quanto à opção referente ao curso de especialização, a mais referida foi a de longa duração na área de reabilitação neuropediátrica (> 30 horas) $(129,50,79 \%)$ (Tabela 1).

Tabela 1. Características demográficas e profissionais.

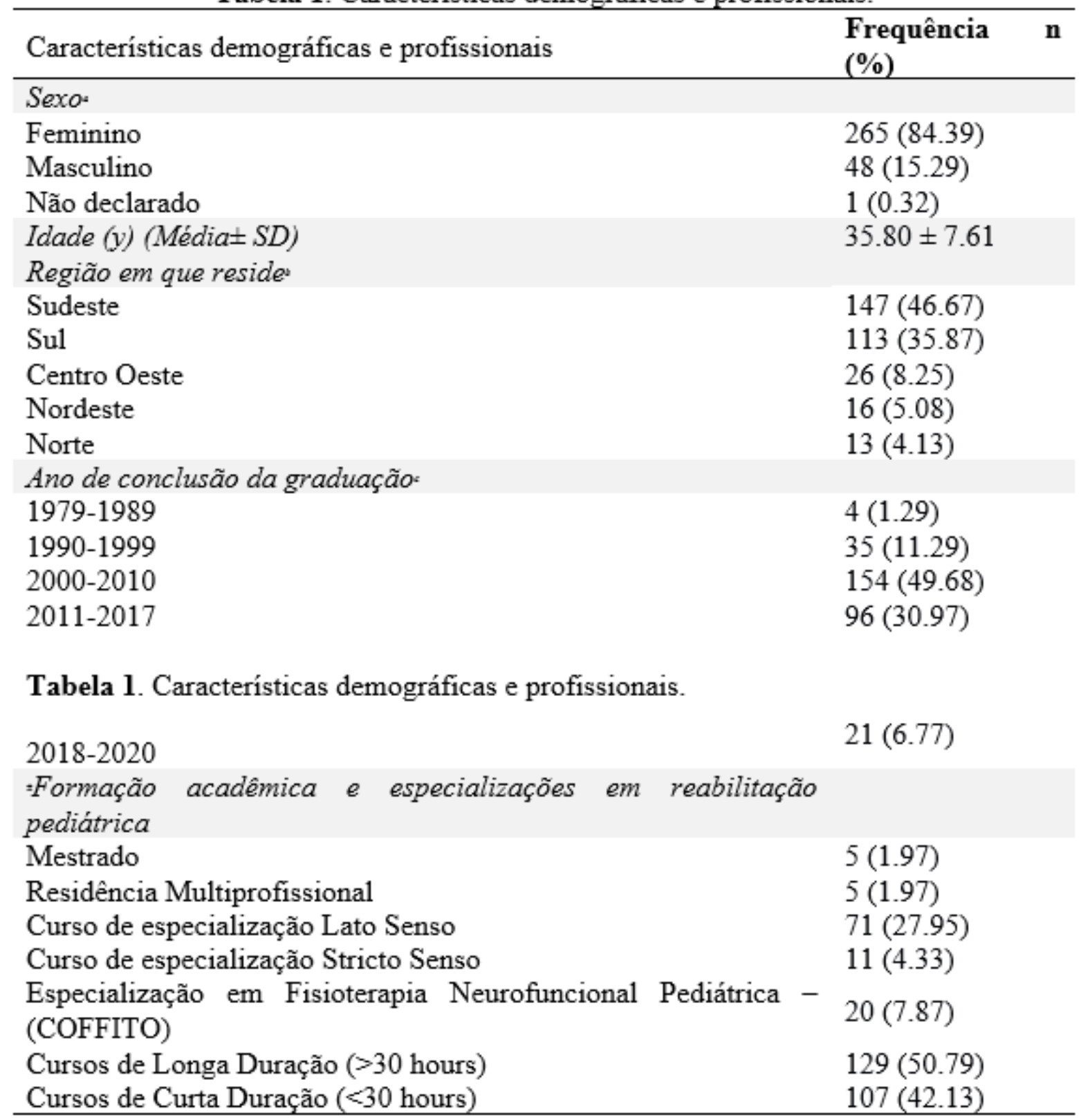

Legendas: 4 omissos, 3 omissos, 8 omissos, \#Categorias que não são mutuamente exclusivas. 


\section{Características do atendimento por telemonitoramento}

Os fisioterapeutas responderam que anteriormente ao surto de Covid-19, a maioria deles atendia mais de 30 crianças semanalmente $(112,35,44 \%)$, dado esse que sofreu alteração durante a pandemia, reduzindo-o para a faixa de 6 a 10 crianças $(68,21,38 \%)$. Além disso, os resultados quanto aos honorários demonstram manutenção do valor cobrado pela maioria dos fisioterapeutas (194, 77,60\%) (Tabela 2).

Referente ao número de sessões e duração dos atendimentos com telemonitoramento, a maioria dos fisioterapeutas realizou uma sessão semanal $(213,67,19 \%)$, com duração entre 11 e 20 minutos (103, 32,39\%), associando, na sua maioria, os métodos sincrônicos e assincrônicos $(145,45,60 \%)$. Além desses achados, a maior parte dos profissionais utilizaram como meio de entrega a troca de mensagens eletrônicas (144, 45,28\%) (Tabela 2).

Quanto ao questionamento de quais os responsáveis por auxiliar as crianças durante o atendimento por telemonitoramento, as mães frequentemente foram mais citadas $(312,98,11 \%)$. O instrumento mais utilizado pelos fisioterapeutas durante as sessões por telemonitoramento foi o uso de bolas, rolos e bancos $(217,69,11 \%)$. Juntamente a essas questões, tratando-se de aspectos pertinentes às dificuldades abordadas pelos fisioterapeutas durante a sessão de telemonitoramento, os campos mais reportados foram a falta de compreensão por pais ou responsáveis em manuseios específicos $(139,44,55 \%)$ e problemas de conexão com a internet $(50,16,03 \%)$ (Tabela 2).

A apresentação de conferência ou reunião referente a utilização do método telemonitoramento foi relatada como existente pela maioria dos fisioterapeutas $(219,69,30 \%)$. Entretanto, apesar da necessidade momentânea do uso do atendimento a distância, a maioria dos entrevistados não demonstram pretensão em utilizar o telemonitoramento após a pandemia, mesmo com a autorização legal existente para isso $(175,55,21 \%)$ (Tabela 2$)$.

Tabela 2. Perfil da assistência em telerreabilitação

\begin{tabular}{lc}
\hline Perguntas referentes a assistência e entrega do telemonitoramento & $\begin{array}{l}\text { Frequência } \\
\mathbf{n}(\%)\end{array}$ \\
\hline Quantas crianças você atendia semanalmente antes da pandemia da Cc \\
19. & $5(1.58)$ \\
$0-2$ crianças & $13(4.11)$ \\
$3-5$ crianças & $30(9.49)$ \\
$6-10$ crianças & $34(10.76)$ \\
$11-15$ crianças & $56(17.72)$ \\
$16-20$ crianças & $66(20.89)$ \\
$21-30$ crianças & $112(35.44)$ \\
$>30$ crianças & \\
Quantas crianças você assistiu semanalmente durante a pandemia da & \\
Covid-19? & $12(3.77)$ \\
$0-2$ crianças & $43(13.52)$ \\
$3-5$ crianças & $68(21.38)$ \\
$6-10$ crianças &
\end{tabular}


$11-15$ crianças

$16-20$ crianças

$21-30$ crianças

$>30$ crianças

Qual foi a duração média de sua assistência por telemonitoramento?

$0-10$ minutos

$11-20$ minutos

$21-30$ minutos

$31-40$ minutos

$41-50$ minutos

$>50$ minutos

Qual $o$ número de sessões semanais por paciente usando telemonitoramento?

$0-1$ sessão

$2-3$ sessões

$4-5$ sessões

$>5$ sessões

Qual opção se ajusta melhor às taxas cobradas pela sessão de telemonitoramento?.

Houve redução do valor cobrado por sessão.

Manutenção do valor cobrado por sessão.

Houve aumento no valor cobrado por sessão.

Qual dos seguintes métodos de entrega do telemonitoramento você mais utilizou com seus pacientes?

Telefonemas

Mensagens (WhatsApp, E-mail, SMS) chamadas de WhatsApp)

Sincrônico

Assincrônico

Ambos foram equivalentes

Houve alguma conferência, palestra ou reunião no seu local de trabalho sobre telemonitoramento?

Sim

Não

\#Você utiliza / utilizou algum instrumento específico como bonecos, bolas ou outros materiais para demonstrar aos responsáveis os exercícios e manuseio dos pacientes? Indique qual deles: 
Bonecas de pano

Brinquedos

Bolas, rolos e bancos

Próprio corpo do terapeuta

Desenhos

Vídeos disponíveis na internet

Outros

\#Quais destes foram responsáveis por ajudar as crianças durante a terapia por telemonitoramento?

Mãe

Pai

Irmãos

Avôs

Tios

Cuidadores

Técnico em Enfermagem

Não houve auxílio de terceiros

Outros

Qual foi a principal dificuldade em utilizar o telemonitoramento?.

Dificuldade de conexão com a internet

Não entendimento dos pais, responsáveis ou cuidadores em manuseios

Mal uso de aplicativos e interfaces de orientação

Não comprometimento dos horários pré-estabelecidos para $\circ \quad 30$ telemonitoramento

Distrações do ambiente

Não disponibilidade de materiais e aparelhos específicos 
pandêmico?s

$\operatorname{Sim}$

Não

Legendas: ${ }^{2} 2$ omissos, ${ }^{\circ} 1$ omisso, 68 omissos, 2 omissos, 6 omissos, ${ }^{1} 1$ omisso; \# Categorias que não são mutuamente exclusivas.

\section{DISCUSSÃO}

A pandemia de Covid-19 representou um desafio para diversas áreas. Na fisioterapia o atendimento não-presencial foi possibilitado para tentar mitigar os efeitos gerados nesse período pandêmico. Aqui reportamos as respostas de 318 fisioterapeutas de diferentes regiões do país, que caracterizam os seus atendimentos por telemonitoramento a crianças com doenças neurológicas. Entre os principais achados, destacamos: decréscimo no número de atendimentos semanais no período da pandemia quando comparado ao período anterior e os telemonitoramentos foram realizados, na sua maioria, uma vez por semana, com duração da sessão entre 11 e 20 minutos, utilizando a combinação das modalidades síncrona e assíncrona. Os fisioterapeutas reportaram que as principais dificuldades durante as sessões por telemonitoramento foram a falta de compreensão dos pais ou responsáveis no manuseio de exercícios e problemas de conexão com a internet. Além disso, a maioria dos profissionais relataram não ter pretensão de adotar o telemonitoramento, caso seja permitido legalmente após a pandemia.

Um dos dados que mais chama a atenção é a diminuição do número de atendimentos semanais. Previamente à pandemia, 112 fisioterapeutas assistiam 30 crianças ou mais por semana, número esse que caiu para 45 durante esse período. Apesar de ser um dado chamativo, já era esperado por duas razões: as medidas de isolamento social e o perfil de crianças atendidas na APAE. As medidas de isolamento e distanciamento social adotadas no Brasil foram seguidas por diversas instituições, dentre elas podemos destacar o fechamento temporário de serviços não essenciais e a diminuição da capacidade de lotação de espaços físicos (OLIVEIRA et al., 2020). Outro ponto importante é que diversas APAEs interromperam os seus atendimentos no início da pandemia e continuaram sua assistência por atendimento remoto. Entretanto, os pacientes atendidos na APAE, por ser uma Organização Não Governamental (ONG), apresentam um perfil econômico mais baixo, o que pode ter gerado situações como ausência de responsável para ajudar no atendimento durante a sessão e problemas com internet, como reportado aqui. $\mathrm{O}$ status econômico talvez explique também a opção por usar a combinação de métodos (síncrono e assíncrono) e a utilização de WhatsApp como principal meio de telemonitoramento, uma vez que esse é o aplicativo de mensagens mais amplamente utilizado no Brasil nas diferentes camadas sociais.

As mães, seguidas dos pais e irmãos, foram as pessoas que mais ajudaram durante as sessões por telemonitoramento. É importante ressaltar que durante a assistência por essa mo- 
dalidade de atendimento é essencial a presença de um responsável adulto pela criança, a fim de auxiliar em exercícios, que muitas vezes não são possíveis de serem realizados pela debilidade física criança e por questões de privacidade e segurança (ZYLSTRA, 2013; THEODOROS, 2008). Em um estudo realizado na Itália durante a pandemia, Provenzi et al., (2020) demonstraram as mesmas atribuições reportadas na presente pesquisa, revelando que as mães eram as principais ajudantes durante a telerreabilitação. Faz-se importante destacar a sobrecarga que algumas dessas mães podem estar passando durante a pandemia. Em contextos normais já se sabe da sobrecarga física e mental que as mães de crianças com paralisia cerebral, principal doença neuropediátrica, passam (VADIVELAN et al., 2020; YANG et al., 2020). Willner et al. (2020) reportaram em um estudo transversal que a sobrecarga de cuidadores de crianças com desordens do desenvolvimento ou de crianças sem nenhuma patologia aumentou 2 a 3 vezes, quando comparado ao período pré-pandemia.

As principais dificuldades manifestadas pelos fisioterapeutas durante as sessões por telemonitoramento foram a má compreensão dos exercícios terapêuticos e problemas com conexão de internet. Esse último é evidenciado como um problema comum em países subdesenvolvidos e em desenvolvimento, sendo reportado por alguns estudos na área de reabilitação. Para Leochico et al., (2020) as principais dificuldades encontradas na implementação da telerreabilitação em um país em desenvolvimento, Filipinas, foram a má qualidade da conexão de internet, ausência de eletricidade e falta de segurança dos dados. Outras revisões sistemáticas explorando os impasses e barreiras da telessaúde também citam problemas correlacionados a esse fator como algo limitante e lembrado pelos usuários e provedores de saúde (ORLANDO et al., 2019; WERNHART et al., 2019).

Aspectos referentes à má compreensão dos exercícios terapêuticos podem estar relacionados à baixa familiarização dos pacientes com a telerreabilitação, como também devido a não experiência prévia dos fisioterapeutas frente a essa modalidade de atendimento. Sanders et al., (2012) em um estudo qualitativo observacional relataram que uma das principais queixas dos pacientes na adoção de um sistema de telessaúde envolvia o não entendimento no uso do equipamento e de algumas instruções passadas por profissionais. De fato, Utidjian e Abramson, (2016), em uma revisão sobre a telessaúde na pediatria ressaltam que entre as principais preocupações dos provedores e pacientes estão a dificuldade técnica e familiaridade com a tecnologia, bem como má compreensão de instruções. Em vista disso, palestras e cursos sobre o uso das diferentes ferramentas que podem ser utilizadas na telerreabilitação, associadas ao treinamento prévio das equipes de assistência, talvez sejam alternativas relevantes para promover um maior entendimento diante da modalidade e sanar objeções que possam dificultar a aplicabilidade do telemonitoramento.

Este estudo contém algumas limitações, como a ausência de cálculo amostral, período curto de recrutamento e o fato de a coleta ter sido restrita apenas a fisioterapeutas da REDE APAE. Entretanto, esse estudo nos fornece informações importantes sobre as características dessa nova modalidade de atendimento fisioterapêutico em crianças com alterações físicas de- 
correntes de lesões neurológicas. Espera-se que os dados dispostos aqui auxiliem esses profissionais e gestores perante a utilização do telemonitoramento como meio de atendimento.

\section{REFERÊNCIAS}

ADERONMU, J. A. Emerging challenges in meeting physiotherapy needs during COVID-19 through telerehabilitation. Bulletin Of Faculty Of Physical Therapy, Cairo, v. 25, n. 1, p. 1-4 dez. 2020. Disponível em: http://dx.doi.org/10.1186/s43161-020-00018-4. Acesso em: 18 nov. 2020 .

AYATOLLAHI, H; SARABI, F. Z; LANGARIZADEH, M. Clinicians' Knowledge and Perception of Telemedicine Technology. Perspect Health Inf Manag, Columbia, v. 12, n. 1, p. 1-6, 2015. Disponível em: https://www.ncbi.nlm.nih.gov/pmc/articles/PMC4632872/. Acesso em: 18 nov. 2020.

CAMDEM, C; SILVA, M. Pediatric Teleheath: Opportunities Created by the COVID-19 and Suggestions to Sustain Its Use to Support Families of Children with Disabilities. Phys Occup Ther Pediatr, v. 41, p. 1-17, out. 2020. Disponível em: https://doi.org/10.1080/01942638.202 0.1825032. Acesso em: 18 nov. 2020.

COFFITO. Resolução $\mathbf{N}^{0}$ 516, de 20 de março de 2020-teleconsulta, telemonitoramento e teleconsultoria. 2020. Disponível em: https:/www.coffito.gov.br/nsite/?p=15825. Acesso em: 18 nov. 2020.

DHOCHAK, N. et al. Pathophysiology of COVID-19: why children fare better than adults? The Indian Journal Of Pediatrics, Calcutá, v. 87, n. 7, p. 537-546, maio 2020. Disponível em: http://dx.doi.org/10.1007/s12098-020-03322-y. Acesso em: 18 nov. 2020.

HAN, E. et al. Lessons learnt from easing COVID-19 restrictions: an analysis of countries and regions in Asia Pacific and Europe. Lancet Microbe, v. 396, p. 1525-1534, nov. 2020. Disponível em: https://doi.org/10.1016/S0140-6736(20)32007-9. Acesso em: 18 nov. 2020.

HULS, A. et al. Medical vulnerability of individuals with Down syndrome to severe COVID-19 - data from the Trisomy 21 Research Society and the UK ISARIC4C survey. E Clinical Medicine, v. 33, p. 1 - 11, 2021. Disponível em: https://doi.org/10.1016/j.eclinm.2021.100769. Acesso em: 01 nov. 2021.

LANGBECKER, Detal.Using survey methodsintelehealth research:A practical guide.JTelemed Telecare, v. 23, p. 770-779, 2017. Disponível em: https://doi.org/10.1177/1357633X17721814. Acesso em: 18 nov. 2020.

LEOCHICO, C. F. D. et al. Challenges to the Emergence of Telerehabilitation in a Developing 
Country: a systematic review. Frontiers In Neurology, v. 11, p. 1-14 set. 2020. Disponível em: http://dx.doi.org/10.3389/fneur.2020.01007. Acesso em: 18 nov. 2020.

MARESCA, G. et al. Tele-Neuro-Rehabilitation in Italy: state of the art and future perspectives. Frontiers In Neurology, v. 11, p. 1-12, set. 2020. Disponível em: http://dx.doi.org/10.3389/ fneur.2020.563375. Acesso em: 18 nov. 2020.

MARINHO, P. R. D et al. Covid-19 in Brazil: a sad scenario. Cytokine \& Growth Factor Reviews, v. 58, p. 51-54, nov. 2020. Disponível em: http://dx.doi.org/10.1016/j.cytogfr.2020.10.010. Acesso em: 18 nov. 2020.

MEIRELES, A. L. F; MEIRELES L. C. F. de. Impact of Social Isolation due to the COVID-19 Pandemic in Patients With Pediatric Disorders: Rehabilitation Perspectives From a Developing Country. Physical Therapy, v. 100, p. 1910-1912, nov. 2020. Disponível em: https://doi. org/10.1093/ptj/pzaa152. Acesso em: 18 nov. 2020.

OLIVEIRA, $\mathrm{M}$ et al. Controlling the COVID-19 pandemic in Brazil: a challenge of continental proportions. Nature Medicine, v. 26, n. 10, p. 1505-1506, set. 2020. Disponível em: http:// dx.doi.org/10.1038/s41591-020-1071-5. Acesso em: 18 nov. 2020.

ORGANIZAÇÃO MUNDIAL DA SAÚDE. Infection prevention and control guidance for long-term care facilities in the context of COVID-19: interim guidance. 2020. Disponível em: https://apps.who.int/iris/handle/10665/331508. Acesso em: 18 nov. 2020.

ORLANDO, J. F; BEARD, M; KUMAR, S. Systematic review of patient and caregivers' satisfaction with telehealth videoconferencing as a mode of service delivery in managing patients' health. Plos One, v. 14, n. 8, p. 1-20 ago. 2019. Disponível em: http://dx.doi.org/10.1371/journal.pone.0221848. Acesso em: 18 nov. 2020.

PEEK, K. et al. Physiotherapists' perceptions of patient adherence to prescribed self-management strategies: a cross-sectional survey of Australian physiotherapists. Disabil Rehabil, v 39, p. 1932-1938, 2017. Disponível em: https://doi.org/10.1080/09638288.2016.1212281. Acesso em: 18 nov. 2020.

PROVENZI, L. et al. Italian parents welcomed a telehealth family-centred rehabilitation programme for children with disability during COVID-19 lockdown. Acta Paediatr, v. 110, n. 1, p. 1-3, out. 2020. Disponível em: https://doi.org/10.1111/apa.15636. Acesso em: 18 nov. 2020.

SANDERS, C. et al. Exploring barriers to participation and adoption of telehealth and telecare within the Whole System Demonstrator trial: a qualitative study. BMC Health Services Research, v. 12, n. 1, p. 1-12, jul. 2012. Disponível em: http://dx.doi.org/10.1186/1472-6963-12220. Acesso em: 18 nov. 2020. 
TENFORDE, A. S. et al. Outpatient Physical, Occupational, and Speech Therapy Synchronous Telemedicine. American Journal Of Physical Medicine \& Rehabilitation, v. 99, n. 11, p. 977-981, ago. 2020. Disponível em: http://dx.doi.org/10.1097/phm.0000000000001571. Acesso em: 18 nov. 2020.

THEODOROS, D; RUSSEL, T. Telerehabilitation: current perspectives. Stud Health Technol Inform, v. 131, p. 191-209, 2008. Disponível em: https://pubmed.ncbi.nlm.nih.gov/18431862/. Acesso em: 18 nov. 2020.

UTIDJIAN, L; ABRAMSON, E. Pediatric Telehealth. Pediatric Clinics Of North America, v. 63, n. 2, p. 367-378, abr. 2016. Disponível em: http://dx.doi.org/10.1016/j.pcl.2015.11.006. Acesso em: 18 de novembro de 2020.

VADIVELAN, K. et al. Burden of caregivers of children with cerebral palsy: an intersectional analysis of gender, poverty, stigma, and public policy. BMC Public Health, v. 20, p. 1-8, 2020. Disponível em: https://doi.org/10.1186/s12889-020-08808-0. Acesso em: 18 nov. 2020.

WERNHART, A.; GAHBAUER, S.; HALUZA, D. EHealth and telemedicine: practices and beliefs among healthcare professionals and medical students at a medical university. Plos One, v. 14, n. 2, p. 1-13, 28 fev. 2019. Disponível em: http://dx.doi.org/10.1371/journal.pone.0213067. Acesso em: 18 nov. 2020.

WILLNER, P. et al. Effect of the COVID-19 pandemic on the mental health of carers of people with intellectual disabilities. J Appl Res Intellect Disabil, Lancashire, v. 33, p. 1523-1533, 2020. Disponível em: https://doi.org/10.1111/jar.12811. Acesso em: 18 nov. 2020.

YANG, H. W. et al. Family Perspectives toward Using Telehealth in Early Intervention. Journal Of Developmental And Physical Disabilities, v. 33, n. 2, p. 197-216, maio 2020. Disponível em: http://dx.doi.org/10.1007/s10882-020-09744-y. Acesso em: 18 nov. 2020.

ZYLSTRA, S. E. Evidence for the Use of Telehealth in Pediatric Occupational Therapy. J Occup Ther Sch Early Interv, v. 6, p. 326-355, ago. 2013. Disponível em: https://doi.org/10.108 0/19411243.2013.860765. Acesso em: 18 nov. 2020. 